\title{
Macrophage-Induced Apoptosis Limits Endovascular Trophoblast Invasion in the Uterine Wall of Preeclamptic Women
}

\author{
Frank Reister, Hans-Georg Frank, John C. P. Kingdom, Wolfgang Heyl, \\ Peter Kaufmann, Werner Rath, and Berthold Huppertz \\ Department of Obstetrics and Gynecology (FR, WH, WR) and Department of Anatomy (H-GF, PK, BH), University \\ of Technology, Aachen, Germany; and Division of Maternal/Fetal Medicine (FR, JCPK), Mount Sinai Hospital, \\ University of Toronto, Ontario, Canada
}

\begin{abstract}
SUMMARY: Impaired invasion of uteroplacental arteries by extravillous trophoblast cells is a key pathogenic mechanism of preeclampsia. We previously demonstrated that reduced trophoblast invasion into uteroplacental spiral arteries was associated with an excess of macrophages in and around these arteries. To explore the significance of these observations, we correlated the extent of extravillous trophoblast apoptosis in placental bed biopsy specimens with macrophage distribution and studied the effect of macrophages upon trophoblast apoptosis in vitro. Extravillous trophoblast hybrid cells were cocultured with activated macrophages exposed to exogenous tumor necrosis factor $\alpha$ (TNF $\alpha$ ), anti-tumor necrosis factor receptor I (TNF-RI), and tryptophan depletion, and the rates of trophoblast apoptosis were measured. Extravillous trophoblast hybrid cells showed increased rates of apoptosis following exposure to exogenous $\mathrm{TNF} \alpha$, with tryptophan depletion, and when cocultured with activated macrophages. The proapoptotic effects of macrophages in vitro were completely inhibited only by simultaneous addition of tryptophan and anti-TNF-RI. Our data indicate that macrophages, residing in excess in the placental bed of preeclamptic women, are able to limit extravillous trophoblast invasion of spiral arterial segments through apoptosis mediated by the combination of TNF $\alpha$ secretion and tryptophan depletion. The mechanisms by which macrophages are activated and recruited to the placental bed are presently unknown but are likely central to the pathogenesis of preeclampsia. (Lab Invest 2001, 81:1143-1152).
\end{abstract}

$P$ reeclampsia remains a leading cause of maternal and perinatal morbidity and mortality worldwide. The disorder is thought to result from chronic placental ischemia, as a direct consequence of inadequate transformation of the spiral segments of the uteroplacental arteries by the invading extravillous trophoblast (Brosens et al, 1967, 1972). In recent years a number of studies have defined the molecular mechanisms, intrinsic to extravillous trophoblast, that appear to limit the invasiveness of these cells into the uterine wall (Benirschke and Kaufmann, 2000; Graham and McCrae, 1996; Zhou et al, 1993, 1997).

Recently, we have demonstrated (Reister et al, 1999) an excess of maternal macrophages within and around the walls of poorly transformed spiral arteries in preeclamptic women, which contained little or no extravillous trophoblast. By contrast, spiral artery seg-

Received April 16, 2001.

FR and H-GF contributed equally to this study. The project was supported by the START program of the Medical Faculty, RWTH Aachen, Grant 12/96-S (FR), by the Deutsche Forschungsgemeinschaft, Grants Ka 360/ 7-3 (PK and BH) and Fr 1245/3-1,2 (H-GF and PK, and by IZKF Biomat, Grant TV-G10 (BH).

Address reprint requests to: Dr. Berthold Huppertz, Department of Anatomy, University Hospital Aachen, Wendlingweg 2, D-52057 Aachen, Germany.E-mail:bhuppertz@post.klinikum.rwth-aachen.de ments in normotensive women that were invaded appropriately by extravillous trophoblast were largely devoid of macrophages. The functional significance of these observations is important because either

(a) these macrophages may have been attracted to remove apoptotic extravillous trophoblast cells from the media of the spiral arteries, or

(b) these macrophages may directly induce apoptosis of extravillous trophoblast cells, thereby limiting transformation of these arterial segments.

Macrophages actively influence cells and tissues in their surrounding environment by secreting cytokines (Werb, 1983) such as tumor necrosis factor $\alpha$ (TNF $\alpha)$. Fetally derived macrophages secrete TNF $\alpha$ in vitro, which induces apoptosis via the tumor necrosis factor receptor I (TNF-RI); this receptor is present on villous cytotrophoblast (Yui et al, 1994). The Fas/FasL system is another candidate signaling mechanism for apoptosis. It has been described in both villous and extravillous trophoblast (Hammer et al, 1999). Recent data from our group have revealed that the complete molecular machinery for apoptosis is expressed by villous (Huppertz et al, 1998b) as well as extravillous trophoblast cells (Huppertz et al, 1998a).

In addition, macrophages express and secrete indolamine 2,3 dioxygenase (IDO) (Werner-Felmayer et al, 1989), an enzyme leading to extracellular trypto- 
phan starvation by catabolizing this essential amino acid. In several cell lines, it has been shown that tryptophan starvation induces apoptosis (Konan and Taylor, 1996).

In the present study, the potential interaction between macrophages and extravillous trophoblast cells within spiral arteries was examined using a coculture system to study the induction of extravillous trophoblast apoptosis. In combination with immunohistochemical studies of activated macrophages and extravillous trophoblast apoptosis in placental bed biopsy specimens, our data support a key role for activated macrophages in the induction of apoptosis, and thus limitation of trophoblast invasion, in the placental bed of women with preeclampsia.

\section{Results}

\section{Definitions}

For the purposes of this study, we used the following definitions according to Benirschke and Kaufmann (2000). The placental bed comprises that part of the uterine wall after delivery to which the placenta was attached. It is characterized by an intimate mixture of extravillous trophoblast and decidua or myometrium, respectively. Extravillous trophoblast is defined as all trophoblast cells residing outside placental villi. Interstitial trophoblast comprises the extravillous trophoblast in the placental bed, which had infiltrated the decidual stroma but had not yet invaded the walls of the spiral arteries. Endovascular trophoblast comprises those extravillous trophoblast cells infiltrating the media of uteroplacental arteries and those trophoblast cells replacing the endothelium; both subgroups of endovascular trophoblast are thought to be directly or indirectly derived from the interstitial trophoblast route of invasion (Benirschke and Kaufmann, 2000). Multinucleated giant cells represent the multinucleated subset of interstitial trophoblast cells prevailing in the depth of the placental bed. Their presence was diagnostic of a "placental bed" biopsy.

\section{Immunohistochemistry of Placental Bed Biopsies}

Preeclamptic Patients. The majority of spiral artery segments found in these sections were only poorly invaded by trophoblast. Rather they contained increased numbers of activated macrophages. In the placental bed, interstitial trophoblast cells (immunoreactive for cytokeratin 7; Fig. 1c) in the vicinity of spiral arteries were surrounded by accumulations of macrophages (immunoreactive for CD68; Fig. 1a). This was observed in $64.8 \% \pm 19.7 \%$ of all spiral artery cross sections in the preeclamptic specimens (individual absolute numbers are [spiral artery cross sections/ sections with periarterial trophoblast]: 9/8, 6/4, 5/2, $10 / 7,5 / 4,7 / 3)$. Direct cell-cell contacts between interstitial trophoblast and macrophages were occasionally observed. Ninety-two percent of periarterial interstitial trophoblast cells showed immunoreactivity for TNF-RI (Fig. 1d), and 34\% of periarterial interstitial trophoblast cells showed signs of apoptosis, as proven by cytokeratin 18 neo-epitope formation (M 30 immunoreactivity; Fig. 1b). Forty-two percent of CD 68-positive macrophages surrounding periarterial interstitial trophoblast cells (determined in serial sections) revealed expression of the activation marker 27E10.

Healthy Pregnant Women. In all specimens we detected both well-invaded uteroplacental arteries and noninvaded spiral arteries. Occasionally macrophages were present in the vicinity of spiral arteries. However, accumulations of macrophages accompanying periarterial extravillous trophoblast cells could not be observed in samples of healthy pregnant women.

\section{In Vitro Experiments}

Experiment la (Control). Culture of the choriocarcinoma/extravillous trophoblast hybrid cell line AC$1 \mathrm{M} 59$ in the absence of macrophages resulted in a low (4\%) level of trophoblast apoptosis.

Experiment $\mathrm{lb}$. Culture of AC-1M59 hybrid cells under varying TNF $\alpha$ concentrations $(0.04-25 \mathrm{ng} / \mathrm{ml})$ resulted in a decrease in the numbers of viable trophoblast cells with intact plasma membranes down to $30 \%$, as evidenced by trypan blue exclusion (Fig. 2a). Viability numbers correlated inversely with the concentration of TNF $\alpha$. In parallel, the number of metabolically active hybrid cells (dimethylthiazolyldiphenyltetrazolium bromide [MTT] test) decreased to $34 \%$ (Fig. 2b). Terminal deoxynucleotidyl transferasemediated dUTP nick-end labeling (TUNEL)-positive cells increased from $4 \%$ (without TNF $\alpha$ ) to $12 \%$ (with high doses of TNF $\alpha$ ) (Fig. 2c).

Experiment Ic. Addition of 1 to $5 \mathrm{ng} / \mathrm{ml}$ anti-TNF-RI to experiments performed as in Ib almost completely blocked all effects of exogenous TNF $\alpha$ on viability, metabolic activity, and apoptosis; lower doses of anti-TNF-RI blocked the effects of low doses of TNF $\alpha$ only (Fig. 2, a to c).

Experiment Id. Depletion of tryptophan in the culture medium caused decreasing viability (trypan blue exclusion and MTT test) and increasing apoptosis, with the extent of cell death being inversely related to the tryptophan concentration (Fig. 3).

Experiment Ila. Coculture of macrophages with the trophoblast hybrid cell line AC-1M59 resulted in decreasing viability of hybrid cells. The concentration of macrophages correlated inversely with the numbers of viable hybrid cells, as seen by trypan blue exclusion and MTT test (Fig. 4, a and b). The concentration of macrophages correlated positively with the number of apoptotic hybrid cells as visualized by TUNEL staining (Fig. 4c).

Experiment Ilb. All of the effects of the coculture of hybrid cells with macrophages (ie, decreased number of intact and viable trophoblast hybrid cells, increased incidence of trophoblast apoptosis; see Experiment IIb) could be partially suppressed by supplementing the culture medium with $1 \mathrm{ng} / \mathrm{ml}$ anti-TNF-RI (Fig. 4, a to c).

Experiment I/c. Also addition of 10 to $90 \mu \mathrm{g} / \mathrm{l}$ tryptophan to the culture medium of macrophage/tropho- 

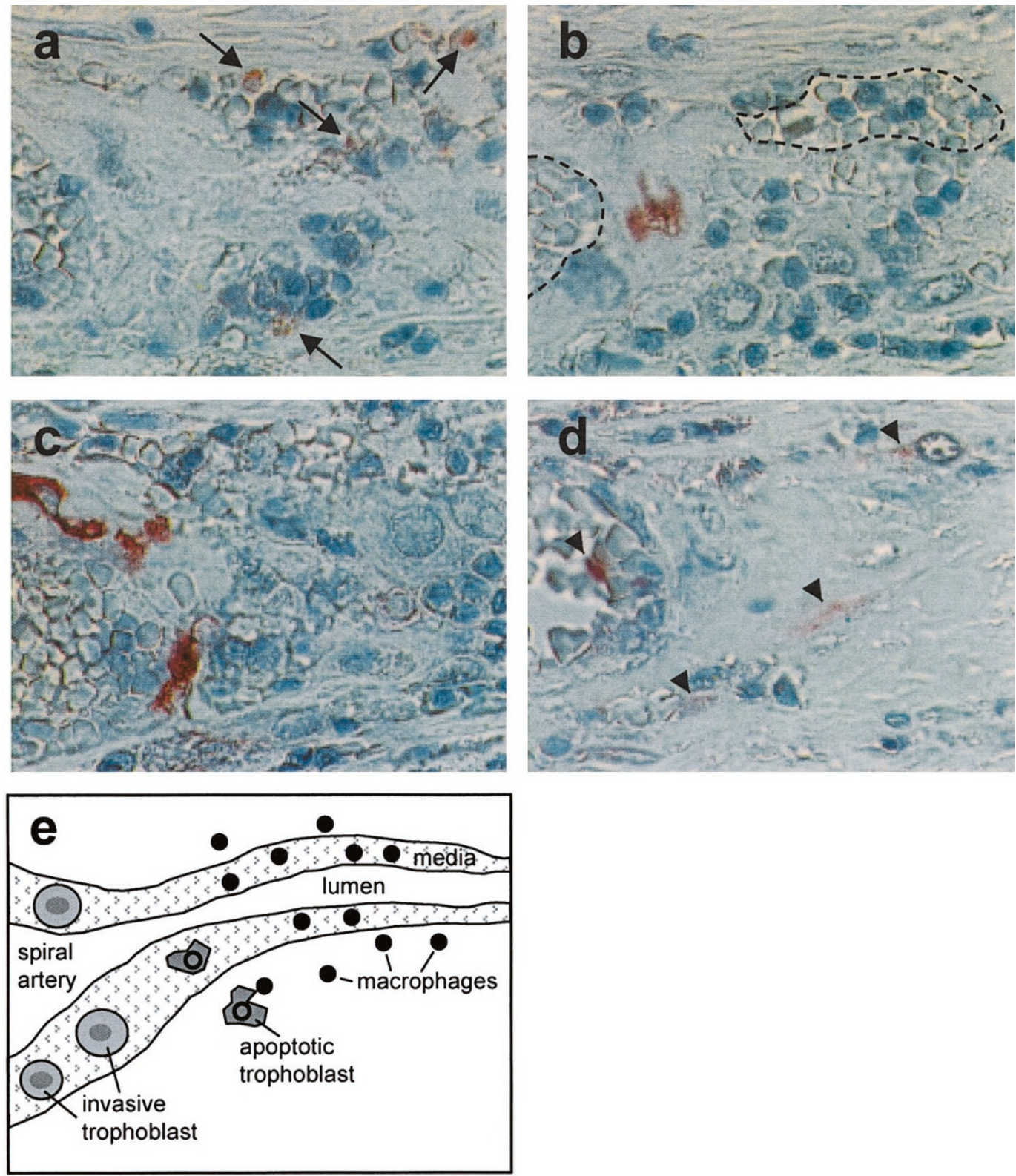

\section{Figure 1.}

Paraffin serial sections from a placental bed biopsy of a preeclamptic patient, tangentially across the wall of a spiral artery at the transition from an undilated (right) to a dilated segment (left). The arterial lumen is outlined by a dotted line in Panel b. Anti-CD 68 (a) reveals an accumulation of macrophages (arrows) in the direct neighborhood of the lumen of the undilated part of the spiral artery. By contrast, in the wall of the dilated segment, cytokeratin 7-positive extravillous trophoblast cells (c), but no macrophages (a), can be found. With M30 (b) the apoptotic subset of extravillous trophoblast can be detected. Anti-tumor necrosis factor receptor I (anti-TNF-RI) staining (d) shows TNF-receptor expression (arrowheads) of the interstitial trophoblast cells. e, Schematic simplification of the findings: reciprocal distribution of extravillous trophoblast cells (dilated segment) and macrophages (narrow, noninvaded segment). Magnification, $\times 1260$.

blast hybrid cocultures only partially reversed the proapoptotic effects of macrophages (Fig. 5).

Experiment IId. Only the addition of both $1 \mathrm{ng} / \mathrm{ml}$ anti-TNF-RI and 30 to $90 \mu \mathrm{g} / \mathrm{l}$ tryptophan to the macrophage/trophoblast hybrid cell cocultures completely blocked the proapoptotic effects of macrophages on trophoblast hybrid cells (Fig. 5).

Experiment Ile (Control). Replacing the trophoblast cell hybrids with their tumor-parent choriocarcinoma cell line AC1-1 in coculture with macrophages did not exert any effect on the trophoblast-derived tumor cells with respect to viability or apoptosis rate (data not shown).

\section{Discussion}

In the present study, we combined two different approaches to investigate the interactions between macrophages and extravillous trophoblast apoptosis during impaired endovascular trophoblast invasion in preeclampsia.

(a) We studied the topographical relations between vital and apoptotic extravillous trophoblast cells with their expression of TNF-RI, as well as with the distribution of activated macrophages in placental bed specimens of normal and preeclamptic pregnancies. 
(a) viable cells, verified by trypan blue exclusion [\%]

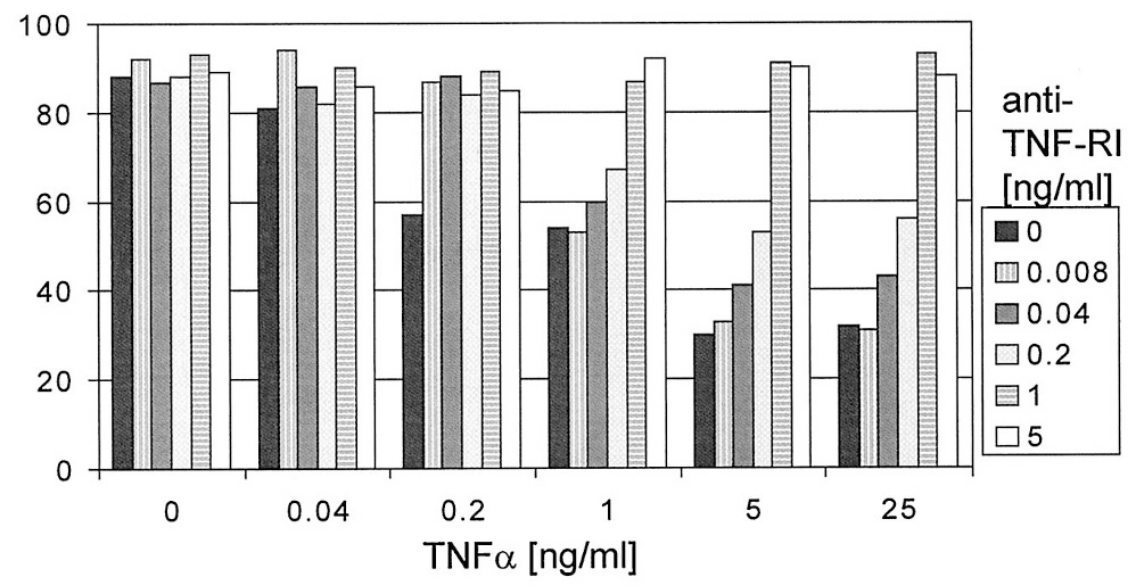

(b) metabolically active, viable cells; MTT test [\%]

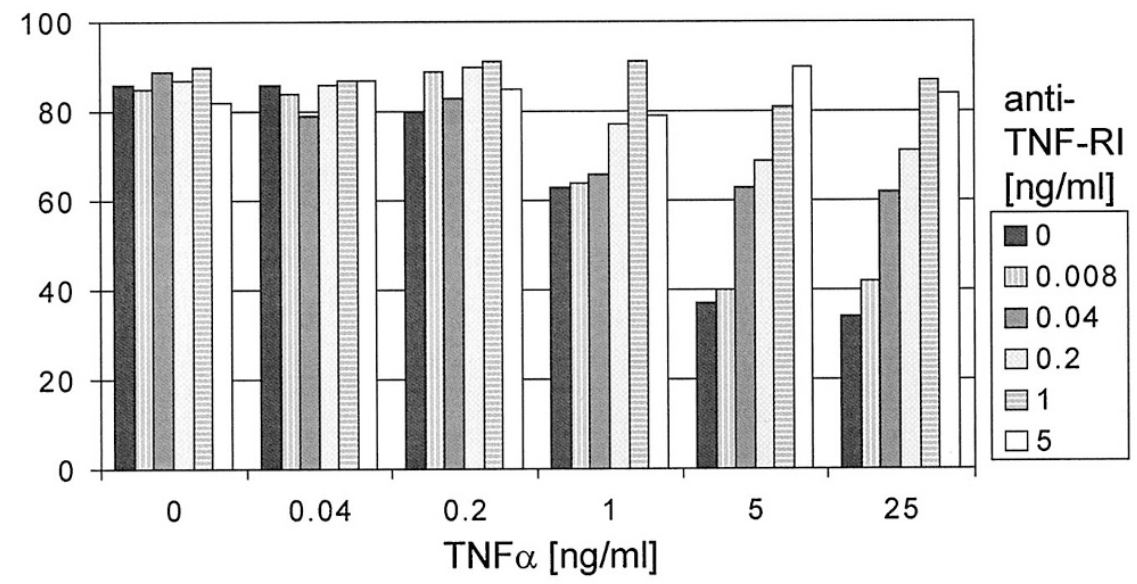

(c) late apoptotic, TUNEL positive cells [\%]

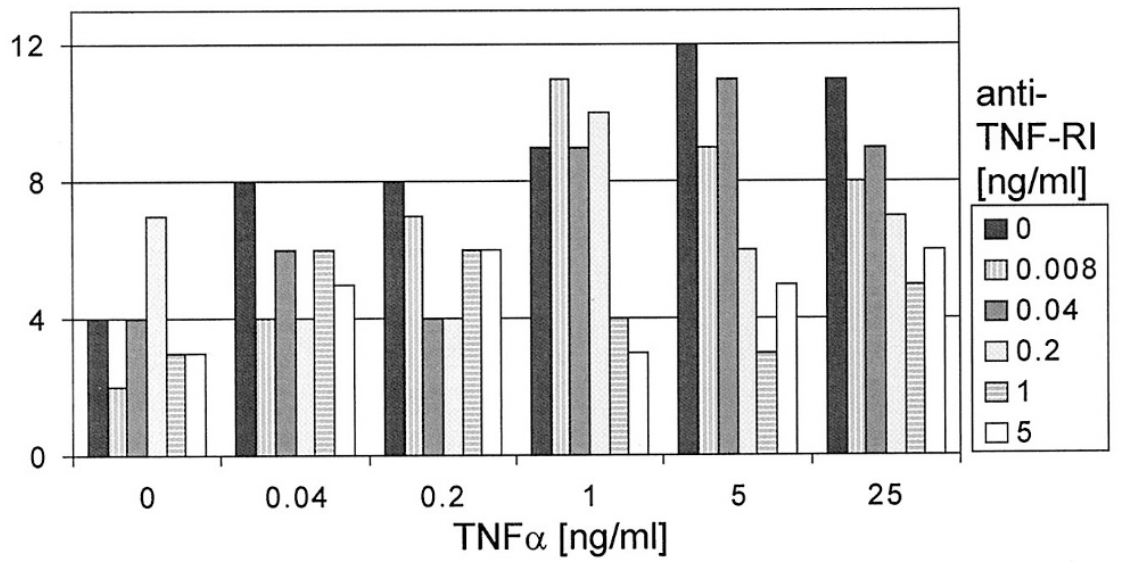

Figure 2.

Effects of increasing concentrations of both tumor necrosis factor $\alpha$ (TNF $\alpha)$ and anti-TNF-RI on trophoblast hybrid cells. a, Percentage of viable cells tested by trypan blue exclusion. b, Percentage of metabolically active, viable cells tested by the dimethylthiazolyl-diphenyltetrazolium bromide (MTT) test. c, Incidence of apoptosis tested by the terminal deoxynucleotidyl transferase-mediated dUTP nick-end labeling (TUNEL) test. Note that the percentage of viable and metabolically active cells decreases with increasing concentrations of TNF $\alpha$ and that this effect can be completely inhibited by addition of anti-TNF-RI. The incidence of trophoblast apoptosis reveals analogous, but inverse, relations. 


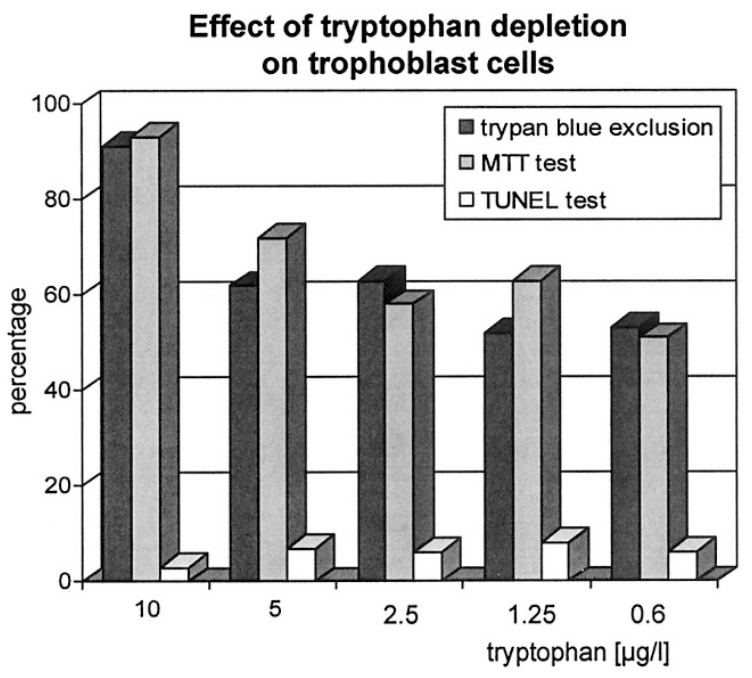

Figure 3.

The culture of extravillous trophoblast hybrid cells under varying concentrations of tryptophan. Tryptophan starvation leads to reduced numbers of both trypan blue-negative (viable) cells, and MTT-positive (metabolically active) cells. By contrast, the incidence of apoptotic, TUNEL-positive trophoblast hybrid cells slightly increases.

(b) We investigated the capacity of macrophages to induce apoptosis in extravillous trophoblast hybrid cells in vitro. Primary extravillous trophoblast cells are of only limited value for in vitro induction of apoptosis because they spontaneously undergo apoptosis shortly after isolation (Gaus et al, 1997). Therefore, we employed a hybrid cell line, generated by hybridization of extravillous trophoblast cells with choriocarcinoma cells (Frank et al, 2000), to address our hypothesis. Different from their choriocarcinoma parent cells (see the control experiments la and Ile), these trophoblast hybrid cells respond to proapoptotic stimuli in vitro as normal extravillous trophoblast cells do in vivo (Huppertz et al, 1998a, and pilot data of this study). Moreover, they share other phenotypic similarities with extravillous trophoblast, such as expression of cytokeratin 7 and human leukocyte antigen (HLA)-G (A. King, Cambridge University, personal communication, 2001) and invasiveness in vitro (G. Desoye, University of Graz, personal communication, 2001).

\section{In Normal and Preeclamptic Pregnancies, Endovascular Trophoblast and Activated Macrophages Show a Reciprocal Distribution Pattern in the Walls of Uteroplacental Arteries}

To date there is a paucity of information regarding the potential interactions between invading extravillous trophoblast cells and maternal macrophages in the placental bed. In our previous study (Reister et al, 1999), we demonstrated a reciprocal distribution of macrophages and extravillous trophoblast cells within and around the walls of uteroplacental spiral arteries in both normal and preeclamptic pregnancies. In healthy pregnant women, dilated spiral artery segments that were invaded by trophoblast cells were largely void of macrophages. By contrast, in preeclamptic women, the outer media of undilated spiral artery segments and the neighboring adventitia were heavily infiltrated by macrophages and generally devoid of extravillous trophoblast. These data support and extend those of earlier investigators (Brosens et al, 1967) who have demonstrated a positive correlation between trophoblast invasion and arterial dilation and a negative correlation between preeclampsia and arterial trophoblast invasion (Brosens et al, 1972; Meekins et al, 1994).

Within the past two years, it has become evident that the complete molecular machinery for apoptosis is present in both the villous and extravillous trophoblast (Huppertz et al, 1998a, 1998b). Moreover, recent data suggesting that apoptosis of extravillous trophoblast is enhanced in preeclampsia (DiFederico et al, 1999; Genbacev et al, 1999) supports the notion that these cells may be lost by programmed cell death. The study by DiFederico et al (1999) was limited to the analysis of placental basal plates from the delivered placenta, rather than from placental bed specimens excised at the time of cesarean section. Accordingly, a complete study of apoptosis during extravillous trophoblast invasion of the uteroplacental arteries was not possible.

Several mechanisms, in addition to apoptosis, have been described to limit extravillous trophoblast invasion into the placental bed. These comprise reduced expression of integrin $\alpha 1 \beta 1$ (Zhou et al, 1993), decreased secretion of matrix metalloproteinase (MMP)-9, and low cell surface plasminogen activator activity (Graham and McCrae, 1996), absent expression of vascular cell adhesion molecule (VCAM)-1, platelet-endothelial cell adhesion molecule (PECAM)-1, and vascular endothelial (VE)cadherin (Zhou et al, 1997) and reduced expression of HLA-G (Hara et al, 1996; Goldman-Wohl et al, 2000). A reduction in the expression of all of these proteins, necessary for extravillous trophoblast differentiation along the invasive pathway, is compatible with the view of progression of extravillous trophoblast along the apoptosis cascade, because it is known that many cells including trophoblast entering the apoptosis cascade down-regulate their general level of transcription (Huppertz et al, 1999).

\section{The Distribution of Activated Macrophages Within and Around the Arterial Walls in Preeclampsia Positively Correlates with Trophoblast Apoptosis in the Vicinity of the Arterial Walls}

We found large numbers of activated macrophages in and beneath most of the spiral arterial walls in preeclampsia, accompanied by increased trophoblast apoptosis in direct vicinity of the vessel walls. These data are supported by a report of Pijnenborg et al (1998), who found a higher incidence of cell clusters secreting $\operatorname{TNF} \alpha$, probably macrophages, in the placental bed of patients with severe forms of preeclampsia. Macrophages could participate in the inhibition of extravillous trophoblast invasion in two ways-passively or actively: 
(a) viable cells, verified by trypan blue exclusion [\%]

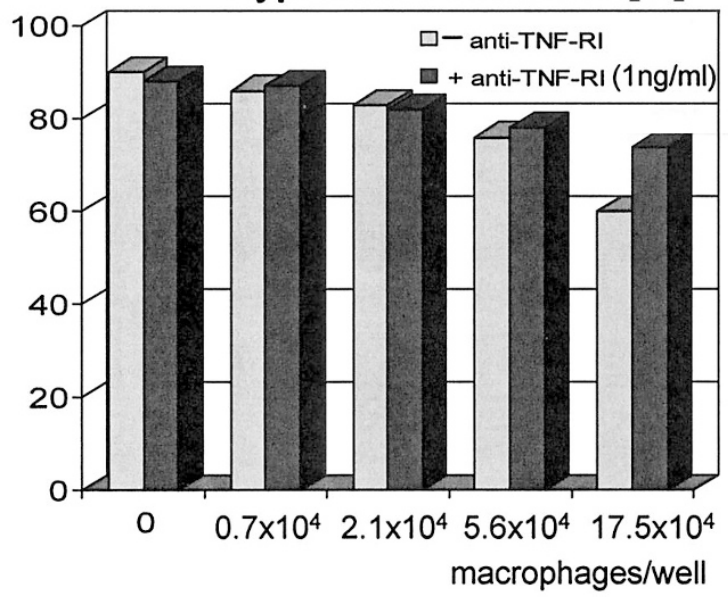

(b) metabolically active, viable cells; MTT test [\%]

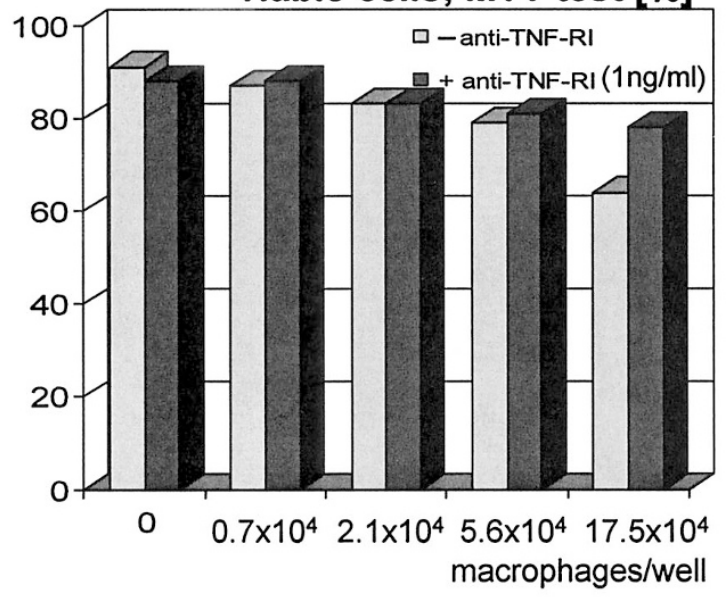

(c) late apoptotic, TUNEL positive cells [\%]

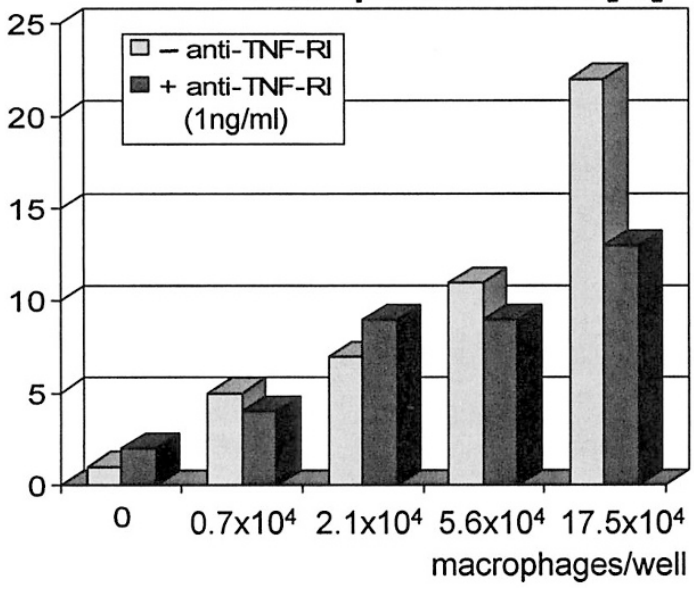

Figure 4.

Coculture of extravillous trophoblast hybrid cells with macrophages in the presence or absence of anti-TNF-RI. In the absence of anti-TNF-RI (light grey bars), increasing numbers of macrophages (a) reduce the percentage of trypan

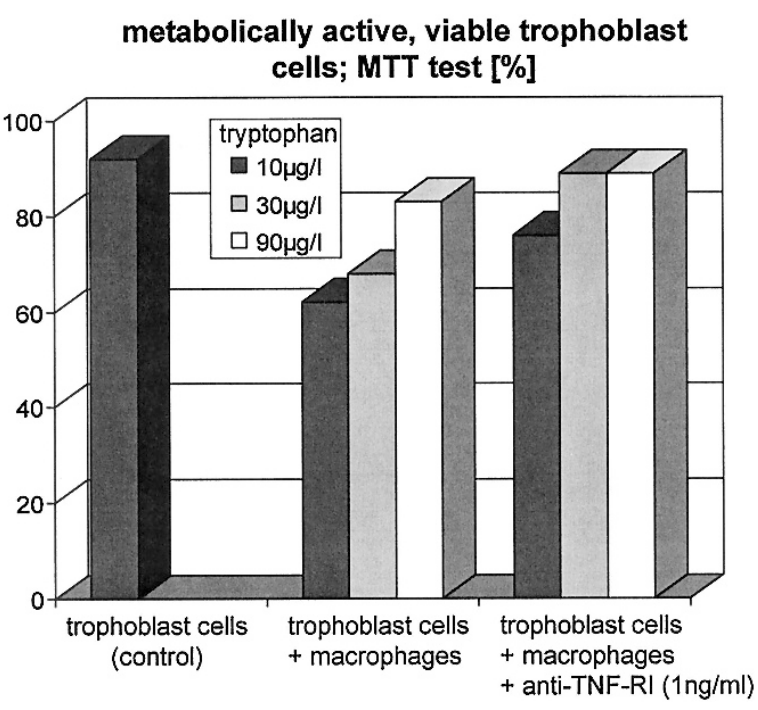

Figure 5.

Cumulative effect of tryptophan supplementation and anti-TNF-RI application on extravillous trophoblast hybrid cells cocultured with macrophages. Hybrid cells were cultured alone (control, left), in the presence of macrophages plus tryptophan (center), and in the presence of macrophages plus tryptophan with the addition of $1 \mathrm{ng} / \mathrm{ml}$ anti-TNF-RI (right). Under control conditions, tryptophan concentrations of $10 \mu \mathrm{g} / \mathrm{l}$ are sufficient to keep the cells viable. When cocultured with macrophages, tryptophan supplementation alone increases viability of trophoblast cells to less than control values. Only supplementation of both, with 30 to $90 \mu \mathrm{g} / \mathrm{l}$ tryptophan and $1 \mathrm{ng} / \mathrm{ml}$ anti-TNF-RI, completely neutralizes the adverse effects of macrophages on trophoblast cells.

(a) passively, because apoptotic extravillous trophoblast cells may attract macrophages;

(b) actively, because macrophages may prevent extravillous trophoblast invasion of the vessel walls by inducing apoptosis in the periarterial interstitial trophoblast.

The latter option is in agreement with our in vitro experiments, which demonstrated that macrophages have the potential to actively induce apoptosis in extravillous trophoblast, and by two distinct mechanisms.

\section{The Apoptosis-Inducing Effects of Exogenous TNFa on Trophoblast Hybrid Cells Can Be Blocked by Application of Anti-TNF-RI In Vitro}

In 1994, Yui and coworkers demonstrated that exogenous TNF $\alpha$ induces trophoblast apoptosis in vitro (Yui et al, 1994). These authors identified TNF-RI as the responsible receptor, which is expressed in villous trophoblast (Yui et al, 1996). Extravillous trophoblast was not studied. We decided to employ immortalized extravillous trophoblast cells generated by hybridization with choriocarcinoma cells (Frank et al, 2000)

blue-negative (viable) trophoblast cells to about two-thirds, (b) reduce the percentage of metabolically active (MTT-test) trophoblast cells to about two-thirds, and (c) increase the incidence of TUNEL-positive trophoblast cells by about 20 -fold. The addition of $1 \mathrm{ng} / \mathrm{ml}$ anti-TNF-Rl (dark grey bars) only partly blocks the adverse effects of macrophages on trophoblast cells, suggesting that TNF $\alpha$ is not the only macrophage-derived stimulus to the induction of apoptosis of trophoblast cells. 
because high rates of spontaneous apoptosis in primary trophoblast-cell isolates limit their use in this model system (Gaus et al, 1997; Huppertz et al, 1999). These hybrid cells exhibited many phenotypic similarities to extravillous trophoblast (Frank et al, 2000), including the expression of TNF-RI. Our in vitro data demonstrate that exogenous TNF $\alpha$ induces apoptosis in extravillous trophoblast hybrid cells. This is in contrast to choriocarcinoma cells, which remain resistant or even use TNF $\alpha$ as a growth factor (Yang et al, 1993; and our own results). The action of TNF $\alpha$ on trophoblast hybrid cells was completely inhibited by the addition of an antibody against TNF-RI, demonstrating that TNF-RI mediates the proapoptotic signal.

\section{Macrophage-Induced Apoptosis in Trophoblast Hybrid Cell Cultures Involves Both Interaction of TNFa with Its Receptor I and Local Depletion of Tryptophan}

Coculture of extravillous trophoblast hybrid cells with activated macrophages induced apoptosis in vitro. The proapoptotic effect of macrophages was only partly reversed by blocking the receptor I of TNF $\alpha$ by antibodies (Fig. 3). This finding suggests that apoptosis induction by macrophages in our coculture system was not exclusively based on TNF $\alpha$ activity.

Macrophages express indole amine 2,3dioxygenase (IDO), a tryptophan-degrading enzyme (Werner-Felmayer et al, 1989). Tryptophan depletion, by the action of IDO, was found to induce apoptosis in certain tumor cell lines (Konan and Taylor, 1996). We, therefore, explored the effects of tryptophan depletion upon apoptosis in our extravillous trophoblast hybrid cells and found an inverse relation between tryptophan concentration in the culture medium and the rate of apoptosis.

Supplementation of macrophage/trophoblast cocultures with tryptophan only partly blocked the proapoptotic effects of macrophages on the trophoblast hybrid cells, whereas simultaneous addition of both tryptophan and anti-TNF-RI inhibited induction of apoptosis to control levels. These data suggest that, in our coculture experiments, macrophage-derived IDO is involved in mediating apoptosis in extravillous trophoblast. Moreover, the tryptophan data explain why blockage of TNF $\alpha$ action alone does not completely inhibit macrophage-induced apoptosis.

Findings by Werner-Felmayer et al (1989) add another interesting aspect: IDO activity is stimulated by interferon (IFN)- $\gamma$, which is known to be secreted by uterine large granular lymphocytes (LGL), the endometrial subclass of natural killer cells (Loke et al, 1995). According to our unpublished findings, this subpopulation of leukocytes is increased in the placental bed of preeclamptic patients, suggesting that tryptophan depletion in vivo also may be involved in impaired trophoblast invasion of the uteroplacental arteries in preeclampsia.

\section{Conclusions}

Our data indicate that macrophage-induced apoptosis of periarterial extravillous trophoblast is involved in impaired endovascular trophoblast invasion in preeclampsia. We suggest that maternal macrophages induce extravillous trophoblast apoptosis by a combination of tryptophan depletion and TNF $\alpha$ secretion in the vicinity of the uteroplacental arteries (Fig. 6). In turn, the apoptotic trophoblast attracts and activates further macrophages, thus providing a feed-forward loop that limits pregnancy-induced transformation of these vessels. Further studies are warranted to explore the biologic basis for macrophage activation in the placental bed of preeclamptic women.

\section{Materials and Methods}

\section{Patients}

Placental bed biopsies were taken during cesarean section from six healthy normotensive women delivering healthy, normally grown infants by primary cesarean section because of breech presentation (gestation: median, 38; range, 38-40 weeks). Normal uterine artery Doppler waveform patterns were demonstrated in each case before delivery. Biopsies were also taken from six women with severe preeclampsia (gestation: median, 31; range, 28-33 weeks), who had diastolic blood pressures repeatedly greater than $100 \mathrm{~mm} \mathrm{Hg}$ and greater than $5 \mathrm{~g}$ per day protein excretion in urine. In addition, these women had evidence of uteroplacental ischemia before delivery, defined as the demonstration of bilateral abnormal uterine artery Doppler waveforms (early diastolic notching and pulsatility index, >1.5) (for review, see Bower et al, 1998). In all six cases of preeclampsia, the babies were small for gestational age (SGA), a condition arbitrarily defined as birthweight less than the 10th percentile, corrected for gestational age. Maternal age (median, 27 years) was similar in each group.

For isolation of peripheral blood monocytes, we used peripheral venous blood from healthy third-

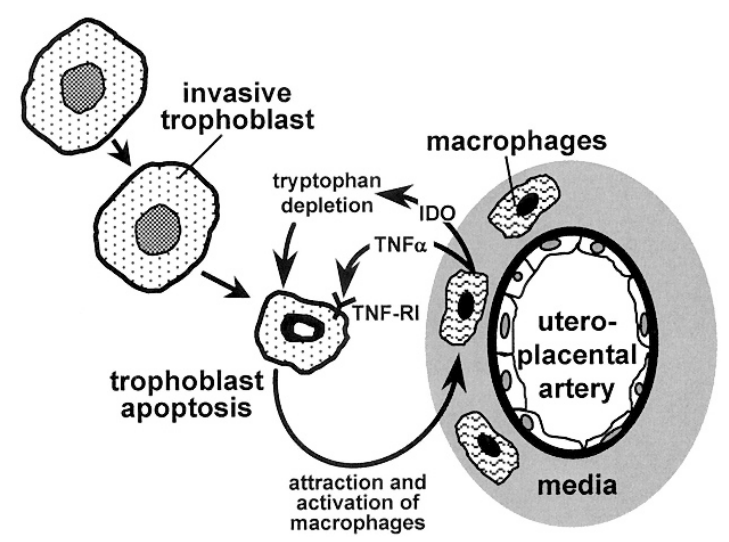

Figure 6.

Schematic representation of the interactions between invasive extravillous trophoblast cells and maternal macrophages in preeclampsia. Our data suggest that activated maternal macrophages induce trophoblast apoptosis around the uteroplacental arteries by means of tryptophan depletion and TNF $\alpha$ secretion. In turn, the apoptotic trophoblast cells attract and activate additional macrophages. It remains unclear how this vicious circle is started in preeclampsia. 
trimester pregnant women not taking any drugs such as corticosteroids.

Our protocol was approved by the Ethics Committee of the Medical Faculty of the University of Technology (EK 512). All women gave written informed consent.

\section{Immunohistochemistry of Placental Bed Biopsies}

Excision and processing of placental bed biopsies were performed as previously described (Reister et al, 1999). Paraffin serial sections $(4 \mu \mathrm{m})$ were used for immunohistochemistry. All biopsies were initially screened by hematoxylin and eosin staining to ensure that the placental bed had been sampled correctly, as defined by the presence of multinucleated trophoblastic giant cells and uteroplacental arteries.

We employed the following primary antibodies on five consecutive sections: (1) a trophoblast marker, anti-cytokeratin 7 (clone OV-TL 12/30; Dako, Hamburg, Germany; 1/200); (2) a marker of late apoptotic trophoblast (clone M 30; Roche, Mannheim, Germany; 1/50) (Kadyrov et al, 2001); (3) a macrophage marker, anti-CD 68 (clone KP-1; Dako; 1/1000); (4) a marker of activated macrophages in early inflammation (clone 27E10; a gift from G. Zwadlo-Klarwasser, Aachen, Germany; 1/100); and, (5) for the detection of the TNF-R1, anti-TNF-RI (clone 16803.1; R\&D Systems, Minneapolis, Minnesota; 1/200). Our immunohistochemical protocol was based on the streptavidinbiotin-technique, "Aachen-Kit" (Frank et al, 1994).

Quantitative evaluation was performed by conventional light microscopy and interference-contrast microscopy. All six cases from each group (control and preeclampsia) were evaluated, and one randomly selected and randomly oriented placental bed biopsy per patient was used for quantification. The percentages listed below are mean values of six sections and related to the total amount of the respective cell type. The following parameters were evaluated:

(a) the absolute number of periarterial extravillous trophoblast cells in a periarterial location: trophoblast cells located within $150 \mu \mathrm{m}$ (roughly two times the diameter of an extravillous trophoblast cell) of the outer limit of the muscular wall of the artery;

(b) the relative number of the trophoblast cells in (a) expressing TNF-RI;

(c) the relative number of the trophoblast cells in (a) showing signs of late apoptosis as indicated by immunoreactivity for $\mathrm{M} \mathrm{30}$; and

(d) the relative number of activated and nonactivated macrophages surrounding the cells in (a). The intercell distance between macrophages and trophoblast cells was determined to be less than 30 microns, the diameter of two macrophages.

\section{Cellular Models}

Choriocarcinoma/Extravillous Trophoblast Hybrid Cells as a Model for Extravillous Trophoblast. Primary extravillous trophoblast cells undergo apoptosis within several hours after isolation without any addi- tional stimulus (Gaus et al, 1997). Therefore, they are not suitable for use in a 24-hour coculture model designed to study induction of apoptosis. This kind of in vitro experiment requires immortalized cell lines with a low background rate of apoptosis. At present, no cell line fulfills all the criteria necessary to be considered extravillous trophoblast (King et al, 2000). The monoclonal choriocarcinoma/extravillous trophoblast hybrid cell line, AC-1M59 (Frank et al, 2000), shares many features with invasive extravillous trophoblast. Among others, it is cytokeratin-positive, exhibits an invasive phenotype in vitro (G. Desoye, University of Graz, personal communication, 2001), and expresses HLA-G (A. King, Cambridge University, personal communication, 2001).

Generation of choriocarcinoma/extravillous trophoblast hybrid cell lines was recently reported in detail (Frank et al, 2000) using the following parental cell lines: (a) AC1-1 cells, HGPRT-negative mutants of the choriocarcinoma cell line JEG-3 (Funayama et al, 1997), and (b) extravillous trophoblast cells isolated from term chorion laeve (Gaus et al, 1997). The fusiongenerated hybrid clone, AC-1M59, proliferates in vitro, exhibits an extravillous phenotype (see above), and expresses TNF-RI as well as TNF-RII (data not shown). AC-1M59 cells were seeded in 24-well plates (Falcon, Cowley, United Kingdom; $2 \times 10^{4}$ cells/well) and cultured for 3 to 4 days until confluence in S-MEM (Gibco, Gaithersburg, Maryland) containing 10\% human serum (blood group $\mathrm{AB}$ ), at $37^{\circ} \mathrm{C}, 100 \%$ humidity, $5 \% \mathrm{CO}_{2}$, and $10 \% \mathrm{O}_{2}$.

Macrophages. Following a series of pilot experiments, we decided to use monocyte-derived macrophages rather than decidual macrophages because the latter revealed a much lower degree of purity and viability compared with blood-derived macrophages (data not shown). As a drawback, one may argue that blood-derived macrophages are not conditioned by the decidual environment. According to Pijnenborg et al (1998), local differences in activation of macrophages within the decidua lead to major differences in cytokine secretion, eg, TNF $\alpha$. Consequently, sampling and extraction of macrophages from large pieces of the decidua would lead to a highly heterogeneous mixture of macrophages from maternal blood and from regions with and without trophoblast invasion. In this situation, we felt that a largely pure population of monocyte-derived macrophages with a well-defined, high degree of activation is superior to a heterogeneous, highly impure, and less viable population of decidual macrophages.

Peripheral blood monocytes were isolated from peripheral venous blood from healthy third-trimester pregnant women by density gradient centrifugation (Ficoll; Seromed, Berlin, Germany) (400 ×g, 40 minutes, $4^{\circ} \mathrm{C}$ ). Mononuclear cells of the interface were carefully collected and washed three times in PBS. Cells of three patients were pooled and the content of monocytes was determined by Giemsa staining and CD14-immunohistochemistry, revealing about 10\% monocytes inside the fraction of mononuclear cells. These cells then were seeded in serial dilutions $(0.2 \times$ 
$10^{5}$ to $5 \times 10^{5}$ monocytes per well) in coculture inserts (Nunc, Roskilde, Denmark) (0.2 $\mu \mathrm{m}$ Anopore membrane) with culture medium (same as for hybrid cells). After 1 hour of culture at $37^{\circ} \mathrm{C}, 100 \%$ humidity, $5 \%$ $\mathrm{CO}_{2}, 10 \% \mathrm{O}_{2}$, supernatant and nonadherent cells (mostly lymphocytes) were discarded and adherent cells were carefully washed. Plating efficiency was about $30 \%$ to $35 \%$. After another 24 hours, cells were used for cocultures. This isolation protocol resulted in greater than $90 \%$ purity and greater than $90 \%$ viability of macrophages, as revealed by Giemsa staining and immunocytochemistry using anti-CD 68. Of those macrophages with greater than $90 \%$ purity, about 95\% showed an activated phenotype as revealed by a positive reaction with the monoclonal antibody 27E10. Pilot experiments revealed that the blood group of macrophage donors did not influence the outcome of the experiments (data not shown).

\section{In Vitro Experiments}

Effects of Soluble Factors on Trophoblast Hybrid Cells. The culture of trophoblast hybrid cells in the absence of macrophages was performed to investigate the effects of exogenous TNF $\alpha$ as well as of tryptophan depletion upon cell viability and apoptosis. The duration of culture was 24 hours. The experiments were performed in triplicate and revealed an intraexperimental error of less than $5 \%$ for trypan blue exclusion and MTT, and less than $2 \%$ for the TUNEL test. Trophoblast hybrid cells were incubated as follows:

Experiment la (control): in S-MEM (Gibco) containing $10 \%$ human serum, without addition of TNF $\alpha$ or depletion of tryptophan;

Experiment lb: with $0.04,0.2,1.0,5.0$, and $25 \mathrm{ng} / \mathrm{ml}$ TNF $\alpha$ (Sigma);

Experiment Ic: with TNF $\alpha$ as in Experiment Ib, but with additional $0.0,0.008,0.04,0.2,1.0$, and $5.0 \mathrm{ng} / \mathrm{ml}$ anti-TNF-RI (Clone No. 16803.1, R\&D Systems); and

Experiment Id: in tryptophan-deficient medium (Select-Amine kit, Gibco) supplemented with tryptophan $(0.6,1.25,2.5,5.0$, and $10 \mu \mathrm{g} / \mathrm{l} ; \mathrm{Gibco})$.

Cocultures of Trophoblast Hybrid Cells with Macrophages. Co-cultures were performed by placing the coculture inserts, containing macrophages, within the 24-well plates containing a near-confluent layer of trophoblast hybrid cells. Before coculture, the culture medium was changed. The duration of coculture was 24 hours, following which the macrophages were discarded and trophoblast hybrid cells were used for the measurements described below. All experiments were done in triplicate and revealed an intraexperimental error of less than $5 \%$ for trypan blue exclusion and $\mathrm{MTT}$, and less than $2 \%$ for the TUNEL test. Cocultures were performed as follows:

Experiment Ila: with trophoblast hybrid cells plus macrophages. The trophoblast hybrid cells were cocultured with $0,0.7 \times 10^{4}, 2.1 \times 10^{4}, 5.6 \times 10^{4}$, and $17.5 \times 10^{4}$ macrophages per well.

Experiment Ilb: with trophoblast hybrid cells plus macrophages supplemented with anti-TNF-RI. Cocul- tures, as in Experiment Ila, were supplemented with 1 $\mathrm{ng} / \mathrm{ml}$ anti-TNF-RI.

Experiment Ilc: with trophoblast hybrid cells plus macrophages supplemented with tryptophan. Cocultures of trophoblast hybrid cells with macrophages $\left(17.5 \times 10^{4}\right)$ were supplemented with 10,30 , or 90 $\mu \mathrm{g} / \mathrm{l}$ tryptophan.

Experiment Ild: with trophoblast hybrid cells plus macrophages supplemented with both, anti-TNF-RI and tryptophan. Cocultures, as in Experiment Ilc (tryptophan supplementation), were additionally supplemented with $1 \mathrm{ng} / \mathrm{ml}$ anti-TNF-RI.

Experiment Ile (control): with AC1-1 choriocarcinoma cells plus macrophages. AC1-1 choriocarcinoma cells (parental cells from the trophoblast hybrid cells) were used for the control experiments in coculture with macrophages because they do not respond to macrophages and TNF $\alpha$.

Evaluation of Coculture Effects and Effects of Soluble Factors. The viability of trophoblast hybrid cells was tested in two ways: (a) integrity of plasma membranes was tested by trypan blue exclusion, and (b) metabolic activity of cells was determined by the MTT assay, in which reduction of tetrazolium bromide to insoluble formazan crystals by mitochondrial activity is measured photometrically. For determination of numbers of trypan blue- or MTT-positive cells, we performed pilot experiments in which known cell counts and photometer readings were plotted against each other and the relation between both was determined (data not shown).

To detect trophoblast hybrid cells which had completed apoptosis, we applied acetone fixation for 10 minutes at $4^{\circ} \mathrm{C}$, followed by the TUNEL test. The protocol for the TUNEL test (TdT-FragEL kit; Calbiochem, San Diego, California) adapted for trophoblast cells was recently reported in detail (Huppertz et al, 1998b, 1999). One hundred cells were counted, both at the margin and in the center of the well, and the proportion of TUNEL-positive cells was calculated.

\section{Acknowledgements}

The technical assistance of Mrs. Lian Shen is gratefully acknowledged. The authors thank Dr. Patrick Bose for critically reading the manuscript.

\section{References}

Benirschke K and Kaufmann P (2000). Pathology of the human placenta, 4th ed. New York: Springer, 171-272.

Bower S, Kingdom J, and Campbell S (1998). Objective and subjective assessment of abnormal uterine artery Doppler flow velocity waveforms. Ultrasound Obstet Gynecol 12: 260-264.

Brosens I, Robertson WB, and Dixon HG (1967). The physiological response of the vessels of the placental bed to normal pregnancy. J Pathol Bacteriol 93:569-579.

Brosens IA, Robertson WB, and Dixon HG (1972). The role of the spiral arteries in the pathogenesis of preeclampsia. Obstet Gynecol Annu 1:177-191. 
DiFederico E, Genbacev O, and Fisher SJ (1999). Preeclampsia is associated with widespread apoptosis of placental cytotrophoblasts within the uterine wall. Am J Pathol 155: 293-301.

Frank HG, Gunawan B, Ebeling-Stark I, Schulten HJ, Funayama $\mathrm{H}$, Cremer U, Huppertz B, Gaus G, Kaufmann P, and Fuzesi $L$ (2000). Cytogenetic and DNA-fingerprint characterization of choriocarcinoma cell lines and a trophoblast/ choriocarcinoma cell hybrid. Cancer Genet Cytogenet 116: $16-22$.

Frank HG, Malekzadeh F, Kertschanska S, Crescimanno C, Castellucci M, Lang I, Desoye G, and Kaufmann P (1994). Immunohistochemistry of two different types of placental fibrinoid. Acta Anat 150:55-68.

Funayama H, Gaus G, Ebeling-Stark I, Takayama M, Fuzesi L, Huppertz B, Kaufmann P, and Frank HG (1997). Parent cells for trophoblast hybridization II: AC1 and related trophoblast cell lines, a family of HGPRT-negative mutants of the choriocarcinoma cell line JEG-3. Trophoblast Res 10:191201.

Gaus G, Funayama H, Huppertz B, Kaufmann P, and Frank HG (1997). Parent cells for trophoblast hybridization I: Isolation of extravillous trophoblast cells from human term chorion laeve. Trophoblast Res 10:181-190.

Genbacev O, DiFederico E, McMaster M, and Fisher SJ (1999). Invasive cytotrophoblast apoptosis in pre-eclampsia. Hum Reprod 14:59-66.

Goldman-Wohl DS, Ariel I, Greenfield C, Hochner-Celnikier D, Cross J, Fisher S, and Yagel S (2000). Lack of human leukocyte antigen-G expression in extravillous trophoblasts is associated with pre-eclampsia. Mol Hum Reprod 6:88-95.

Graham CH and McCrae KR (1996). Altered expression of gelatinase and surface-associated plasminogen activator activity by trophoblast cells isolated from placentas of preeclamptic patients. Am J Obstet Gynecol 175:555-562.

Hammer A, Blaschitz A, Daxbock C, Walcher W, and Dohr G (1999). Fas and Fas-ligand are expressed in the uteroplacental unit of first-trimester pregnancy. Am J Reprod Immunol 41:41-51.

Hara N, Fujii T, Yamashita T, Kozuma S, Okai T, and Taketani $Y$ (1996). Altered expression of human leukocyte antigen $G$ (HLA-G) on extravillous trophoblasts in preeclampsia: Immunohistological demonstration with anti-HLA-G specific antibody "87G" and anti-cytokeratin antibody "CAM5.2." Am J Reprod Immunol 36:349-358.

Huppertz B, Frank HG, and Kaufmann P (1998a). Apoptosis along the invasive trophoblastic pathway. Placenta 19:A35.

Huppertz B, Frank HG, Kingdom JC, Reister F, and Kaufmann $P$ (1998b). Villous cytotrophoblast regulation of the syncytial apoptotic cascade in the human placenta. Histochem Cell Biol 110:495-508.

Huppertz B, Frank HG, Reister F, Kingdom J, Korr H, and Kaufmann $P$ (1999). Apoptosis cascade progresses during turnover of human trophoblast: Analysis of villous cytotrophoblast and syncytial fragments in vitro. Lab Invest 79: 1687-1702.
Kadyrov M, Kaufmann P, and Huppertz B (2001). Expression of a cytokeratin 18 neo-epitope is a specific marker for trophoblast apoptosis in human placenta. Placenta 22:4448.

King A, Thomas L, and Bischof P (2000). Cell culture models of trophoblast II: Trophoblast cell lines: A workshop report. Placenta 14(Suppl A):S113-S119.

Konan KV and Taylor MW (1996). Treatment of ME180 cells with interferon-gamma causes apoptosis as a result of tryptophan starvation. J Interferon Cytokine Res 16:751-756.

Loke YW, King A, and Burrows TD (1995). Decidua in human implantation. Hum Reprod 10:14-21.

Meekins JW, Pijnenborg R, Hanssens M, McFadyen IR, and van Assche A (1994). A study of placental bed spiral arteries and trophoblast invasion in normal and severe pre-eclamptic pregnancies. Br J Obstet Gynaecol 101:669-674.

Pijnenborg R, McLaughlin PJ, Vercruysse L, Hanssens M, Johnson PM, Keith JC, and van Assche FA (1998). Immunolocalization of tumour necrosis factor-alpha (TNF-alpha) in the placental bed of normotensive and hypertensive human pregnancies. Placenta 19:231-239.

Reister F, Frank HG, Heyl W, Kosanke G, Huppertz B, Schröder W, Kaufmann P, and Rath W (1999). The distribution of macrophages in spiral arteries of the placental bed in pre-eclampsia differs from that in healthy patients. Placenta 20:229-233.

Werb $Z$ (1983). How the macrophage regulates its extracellular environment. Am J Anat 166:237-256.

Werner-Felmayer G, Werner ER, Fuchs D, Hausen A, Reibnegger G, and Wachter H (1989). Tumour necrosis factoralpha and lipopolysaccharide enhance interferon-induced tryptophan degradation and pteridine synthesis in human cells. Biol Chem Hoppe Seyler 370:1063-1069.

Yang Y, Yelavarthi KK, Chen HL, Pace JL, Terranova PF, and Hunt JS (1993). Molecular, biochemical, and functional characteristics of tumor necrosis factor-alpha produced by human placental cytotrophoblastic cells. J Immunol 150:5614.

Yui J, Garcia-Lloret M, Wegmann TG, and Guilbert LJ (1994). Cytotoxicity of tumour necrosis factor-alpha and gammainterferon against primary human placental trophoblasts. Placenta 15:819-835.

Yui J, Hemmings D, Garcia-Lloret M, and Guilbert LJ (1996). Expression of the human p55 and p75 tumor necrosis factor receptors in primary villous trophoblasts and their role in cytotoxic signal transduction. Biol Reprod 55:400-409.

Zhou Y, Damsky CH, Chiu K, Roberts JM, and Fisher SJ (1993). Preeclampsia is associated with abnormal expression of adhesion molecules by invasive cytotrophoblasts. J Clin Invest 91:950-960.

Zhou Y, Damsky CH, and Fisher SJ (1997). Preeclampsia is associated with failure of human cytotrophoblasts to mimic a vascular adhesion phenotype. One cause of defective endovascular invasion in this syndrome? J Clin Invest 99:21522164. 